Please do not remove this page

RMIT

UNIVERSITY

\title{
Acupuncture analgesia for temporal summation of experimental pain: A randomised controlled study
}

Zheng, Zhen; Feng, Jian; Da Costa, Cliff; Li, Chun; Li, Shaoyi; Xue, Charlie

https://researchrepository.rmit.edu.au/esploro/outputs/9921861322001341/filesAndLinks?institution=61RMIT_INST\&index=null

Zheng, Z., Feng, J., Da Costa, C., Li, C., Li, S., \& Xue, C. (2010). Acupuncture analgesia for temporal summation of experimental pain: A randomised controlled study. European Journal Of Pain, 14(7), 725-731. https://doi.org/10.1016/j.ejpain.2009.11.006

Document Version: Accepted Manuscript

Published Version: https://doi.org/10.1016/j.ejpain.2009.11.006

Repository homepage: https://researchrepository.rmit.edu.au

(c) 2009 European Federation of International Association for the Study of Pain Chapters. Published by Elsevier Ltd. All rights reserved.

Downloaded On 2023/04/26 23:16:08 +1000 
Thank you for downloading this document from the RMIT Research Repository.

The RMIT Research Repository is an open access database showcasing the research outputs of RMIT University researchers.

RMIT Research Repository: http://researchbank.rmit.edu.au/

\section{Citation:}

Zheng, Z, Feng, J, Da Costa, C, Li, C, Lu, D and Xue, C 2010, 'Acupuncture analgesia for temporal summation of experimental pain: A randomised controlled study', European Journal Of Pain, vol. 14, no. 7, pp. 725-731.

See this record in the RMIT Research Repository at:

http://researchbank.rmit.edu.au/view/rmit:4914

Version: Accepted Manuscript

Copyright Statement: (c) 2009 European Federation of International Association for the Study of Pain Chapters. Published by Elsevier Ltd. All rights reserved.

Link to Published Version:

http://dx.doi.org/10.1016/j.ejpain.2009.11.006 


\title{
Acupuncture analgesia for temporal summation of experimental pain: a randomised controlled study
}

\author{
Authors \\ Zhen Zheng*, Sam Jian Qiang Feng*, Cliff da Costa*, Chun Guang Li*, David Lu*, Charlie Changli Xue* \\ * Traditional and Complementary Medicine Research Program, Health Innovations Research Institute, \& \\ School of Health Sciences, RMIT University, Bundoora, Australia \\ " School of Mathematical and Geospatial Sciences, RMIT University, Bundoora, Australia

$\begin{array}{ll}\text { Correspondent Author: } & \text { Dr Zhen Zheng } \\ & \text { Traditional and Complementary Medicine Research Program } \\ & \text { Health Innovations Research Institute } \\ & \text { School of Health Sciences } \\ & \text { RMIT University, Bundoora, Vic 3083, Australia } \\ & +61399257167 \\ \text { Phone Number: } & +61399257178 \\ \text { Fax Number: } & \text { zhen.zheng@rmit.edu.au }\end{array}$

Category: original article

Keywords: acupuncture, temporal summation of pain, electric stimulation, randomised controlled, temporal effect, spatial effect

No of pages: 23 excluding title page

No of tables: 4

No of figures: 4

Word count:

Abstract: 249

Introduction: 457

The rest: 3977 


\section{Abstract}

Background: Temporal summation of pain, a phenomenon of the central nervous system (CNS), represents enhanced painful sensation or reduced pain threshold upon repeated stimulation. This pain model has been used to evaluate the analgesic effect of various medications on the CNS.

Aims: The present study aimed to evaluate the effects and characteristics of analgesia induced by electroacupuncture (EA), manual acupuncture (MA) and non-invasive shamacupuncture (SA) in healthy humans on temporal summation of pain.

Methods: Thirsty-six pain-free volunteers were randomised into one of the three groups EA $(2 / 100 \mathrm{~Hz})$, MA or SA. Acupuncture intervention was on ST36 and ST40 on the dominant leg delivered by an acupuncturist blinded to the outcome assessment. Both subjects and the evaluator were blinded to the treatment allocation. Pain thresholds to a single pulse (single pain threshold, SPT) and repeated pulses electrical stimulation (temporal summation thresholds, TST) were measured before, 30 minutes after and 24 hours after each treatment.

Results: The baseline values of three groups were comparable. Compared to SA, EA significantly increased both SPT and TST immediately after the treatment on the treatment leg as well as 24 hours after on both the treatment and non-treatment legs (ANOVA, $p<$ 0.05). MA also increased SPT and TST, but the changes were not significantly different from those induced by SA.

Conclusion: EA induces bilateral, segmentally distributed and prolong analgesia on both SPT and TST, indicating a non-centrally specific effect. This effect needs to be verified with heat or mechanical model and in pain patients. 


\section{Introduction}

Acupuncture is commonly used to treat a variety of musculoskeletal pain conditions, including chronic low back pain (Furlan et al. 2005), chronic neck pain (Trinh et al. 2006), osteoarthritis (Berman et al. 2004), tension-type headache (Melchart et al. 2001; Xue et al. 2004), fibromyalgia (Martin et al. 2006) and other painful disorders (Lee 2000; Green et al. 2005). The common features underlying these pains is central sensitisation (Urban and Gebhart 1999). However, there has been no study specifically examining the central inhibitory effects of acupuncture in humans.

Temporal summation of pain, being a central nervous system phenomenon, refers to pain induced by repeated delivery of a non-painful stimulus at a frequency higher than $0.2 \mathrm{~Hz}$ (Price et al. 1978; Arendt-Nielsen et al. 1994). It is understood that repeated afferent inputs gradually enhance activities of the wide dynamic range neurons, i.e., wind up, leading to temporal summation of pain in a psychophysical term. Wind up has been understood to be the precursor of central sensitisation, the main feature underlying all chronic pains (Arendt-Nielsen and Pertersen-Feilx 1995). The activation of N-methyl-Daspartic (NMDA) glutamate receptors is involved in both temporal summation of pain (Price et al. 1978; Price et al. 1994; Arendt-Nielsen et al. 1995) and central sensitisation (Woolf and Thompson 1991).

Temporal summation of pain, therefore, provides an ideal model to study the anti-central sensitisation effect of acupuncture. The $2 \mathrm{~Hz}$ electrical stimulation model developed by Arendt-Nielsen and colleagues (1994) has been successfully used in a number of studies 
in healthy pain-free humans to assess the central inhibitory effects of analgesics, including ketamine (Arendt-Nielsen et al. 1995) - an NMDA antagonist, codeine (Enggaard et al. 2001b)- a weak opioid medication, venlafaxine - an antidepressant (Enggaard et al. 2001a) and isoflurane - a general anaesthetic (Petersen-Felix et al. 1996).

Thus, the first aim of the present study was to investigate the effects of electroacupuncture (EA), manual acupuncture (MA) and sham acupuncture (SA) on temporal summation of pain by measuring the changes of pain thresholds to single pulse electrical stimulation (single pain threshold SPT) and to repeated pulse stimulation (temporal summation pain threshold, TST). In addition, previous studies in healthy humans only tested the immediate effect of acupuncture. However, in the clinic and in animal studies, the effect of one-session acupuncture might last for 24 to 72 hours (Guo et al. 1996b; Fink et al. 2006). Furthermore, acupuncture analgesia has been shown to be widespread in the body. Needling acupoints on one hand can increase pain thresholds on the arm as well as those tested on the face, trunk and leg (Chiang et al. 1973; Zaslawski et al. 2003). The second and third aims of the study were to characterise the duration and spatial distribution of the anti-temporal summation effects of acupuncture treatment. 


\section{Methods}

This randomised, single-blind, sham-acupuncture controlled study was conducted at the Clinical Research Laboratory of RMIT Traditional \& Complementary Medicine (TCM) Research Group with approval from the Human Research Ethics Committee of the RMIT University, Australia. Participants provided written informed consent prior to the commencement of the experiment. They did not receive payment for taking part in the study and were informed that they were free to withdraw from the experiment at any time.

\section{Participants}

Thirty-six acupuncture naïve volunteers aged between 18 and 50 years were recruited via advertisements placed on the University website and posted on campuses. Those who suffered from chronic pain or recurrent pain, took analgesics in the previous two weeks, did not comprehend English, were currently pregnant, had severe heart disease, wore a pacemaker, had a high risk of cardiovascular diseases as assessed using the Cardiovascular Risk Factor Questionnaire, had a brain tumour or epilepsy, had the tendency to bleed, such as being haemophilia, had drug addiction or had skin problems at the needling or testing sites were excluded.

\section{Methods of randomisation and blinding}

Block randomisation was used to ensure that an equal number of participants were allocated into each group. An independent researcher generated the random numbers and allocation sequence using Microsoft Excel software (Microsoft Office 2002, Windows version). The random numbers were individually printed on a piece of paper, which was enclosed in a sealed envelop. Prior to the intervention, the participant picked one envelope 
randomly. The randomisation code was stored in a password-protected computer. The acupuncturist, who delivered EA, MA and SA, was the only person who had access to the codes, and was blinded from outcome assessments. An evaluator blinded to allocation assignment conducted all outcome assessments.

\section{Interventions}

Figure 1 illustrates the sites of acupuncture treatment and pain threshold testing. Acupoints Zusanli (ST36) and Fenglong (ST40) on the dominant leg, called treatment leg in this paper, were used in all three interventions. Sterile single-use acupuncture needles of 0.25 x 40mm (Hwato, Suzhou Medical Appliance Company, China) were used. In the MA group, two needles were inserted to a depth of $20-25 \mathrm{~mm}$. Needles were rotated 180 to 360 degrees between the fingers at $3-5 \mathrm{~Hz}$ in a bidirectional manner, first clockwise then anticlockwise. This stimulation was repeated nine times, and took approximately 10 seconds. De qi sensations, described as soreness, numbness, or distension at the needling site, were produced. This manipulation was repeated every five minutes for six times over a period of 25 minutes as described elsewhere (Zaslawski et al. 2003).

In the EA group, two needles were inserted in the correct depth and manipulated to achieve De qi sensations as described above. The needles were then connected to a modified EA stimulator (Myer 501, Meyer Medical Electronics, Australia) via a pair of alligator clips. Electrical stimuli of square waves with 50 to 70 microseconds of pulse width were delivered at an alternating frequency of 2 and $100 \mathrm{~Hz}$ every 6 seconds. The intensity of stimulation was strong but comfortable with visible muscle contraction. The 
total duration of EA treatment was 25 minutes. The same machine and the mode of EA were used in a previous clinical trial (Zheng et al. 2008).

In the SA group, a modified non-invasive method was used (Lao et al. 1999). A plastic guide tube was tapped at ST36 and ST40 to produce discernible sensation, and then two bent needles, each with a piece of adhesive bandage were then taped to the dermal surface of the two acupoints, respectively, for 25 minutes. Manipulations were made by pressing the bent needles to produce a pressure sensation on the skin surface of the acupoints every five minutes. A non-functioning electrical acupuncture stimulator was connected to the end of the two needles via alligator clips, and was placed on a table within the participants' eyesight, showing a continuously flashing light. De qi sensations were not intended and were avoided.

Participants were treated in a supine position and their vision of the sites of acupuncture needling was blocked by an object placed at their waist level.

\section{Outcome measures}

Primary outcome measures were two types of pain thresholds, i.e., SPT and TST. The pain thresholds were assessed during baseline, immediately after and 24 hours after the interventions at three body sites, namely, the anterior tibia muscle parallel to the midpoint between ST36 and ST40 on both legs and on the dorsal aspect of the non-dominant forearm 30-40 $\mathrm{mm}$ above the wrist crease.

SPT and TST were assessed using the well-documented method (Arendt-Nielsen et al. 1994). Electrical stimulations were delivered with a Grass stimulator (Grass S88, USA) 
that was connected to an isolation unit (Grass SIU5, USA) and a constant current unit (Grass CCU1, USA). The isolation unit was connected between the stimulator and the constant current unit to avoid electricity surge. The constant current unit controlled the magnitude of the electrical current, which was delivered to the skin surface via two adhesive ECG electrodes (1x1 cm diameter, Dantec Medical, USA) filled with gel. A standard pulse train, consisting of five individual 1-ms pulses delivered at $200 \mathrm{~Hz}$ was used as a single pulse stimulus. This single pulse was repeated five times at $2 \mathrm{~Hz}$ to form a train of stimuli. The frequency of the electrical impulses was calibrated with an oscilloscope (HM1007, HAMEG, Germany) before assessing the pain thresholds.

To test SPT, the intensity of the current of a single pulse stimulus was increased from zero milliampere $(\mathrm{mA})$ in steps of $0.2 \mathrm{~mA}$. If no pain was recorded at $2 \mathrm{~mA}$, the current intensity was increased in steps of $0.5 \mathrm{~mA}$ until a pain threshold could be recorded, or a maximum $10 \mathrm{~mA}$ was reached, whichever was reached first. The lowest current value of a single stimulus to elicit a painful sensation, i.e, a definite sharp or pin prick sensation, like an injection as reported by the participant, was recorded as SPT.

TST was tested after SPT. The intensity of the current of a train of stimuli was increased from zero $\mathrm{mA}$ in steps of $0.2 \mathrm{~mA}$. If no TST could be recorded at $2 \mathrm{~mA}$, the current intensity was increased in steps of $0.5 \mathrm{~mA}$ until a TST could be recorded, or a maximum 5 $\mathrm{mA}$ was reached. The lowest current value to elicit a painful sensation in response to the $4^{\text {th }}$ or $5^{\text {th }}$ stimulus of a train of five stimuli was recorded. Both SPT and TST were tested twice. The mean values were recorded as the pain thresholds. Inter-stimulus interval was 30 seconds. 
The secondary outcome measures included Spielberg State and Anxiety Inventory (SSAI) (Spielberg et al. 1983), administered before and after the intervention, and a post-treatment questionnaire, in which the participants were asked to use a verbal pain categorical scale of no pain at all, mild pain, moderate pain and severe pain to rate the sensation of acupuncture treatment. To assess the success of blinding, the participants were asked to state to which group they believed they were allocated to. The evaluator collected the data.

\section{Experimental Procedure}

One day prior to the experiment, the participants were asked not to drink coffee or take any stimulants, and not to miss any meals prior to the experiment. Figure 2 illustrates the experimental procedure. On the experiment day, after obtaining the written consent form, the evaluator prepared the skin area for testing and conducted the 30-minute training session to familiarise the participants with pain induced by electrical stimulation.

After a five-minute rest, the participants entered the baseline SPT and TST assessment phase followed by completing baseline SSAI. After the randomisation, the allocated intervention was given by the acupuncturist. The participant and acupuncturist were free to communicate as if in a clinical situation except any discussion about the study and intervention was avoided. Immediately after the intervention, the participants completed SSAI the $2^{\text {nd }}$ time and the post-treatment questionnaire. At 30 minutes and 24 hours after the intervention, SPT and TST were tested for the $2^{\text {nd }}$ and $3^{\text {rd }}$ times. In total, on the first day, the experiment lasted for 3 hours. The experiment was conducted in a quiet and temperature-controlled room. The room temperature was adjusted to $22-25^{\circ} \mathrm{C}$. 


\section{Statistical analysis}

All statistical analyses were carried out by using the Statistical Program for Social Science (SPSS, version 15.0). The demographic and baseline data were analysed with one-way analysis of variance (ANOVA) or Chi square tests. Paired $t$-tests were used to compare SPT and TST within groups. Intention-to-treat analysis was used for all outcome measures. SPT and TST data were analysed with three-way (intervention group, time, site) ANOVA with two repeated measures (time, site) to detect between intervention group differences across the three time points measured at three body sites. SSAI data were analysed with two-way (intervention group, time) ANOVA with one repeated measure (time) to detect between group differences. Significance level was set at $\alpha=0.05$. Post-hoc one-way ANOVA was used when a statistically significant interaction was shown. Multiple independent-sample t-tests with Bonferroni corrections were also used if there was an intervention effect as shown by one-way ANOVA. Statistical power analysis and sample size calculations were conducted using the MINITAB statistical package (MINITAB, Windows Version 15.0). 


\section{Results}

\section{Baseline comparability}

There were 12 participants in each intervention group and all completed the study. The participants were University students, including 21 males and 15 females. The average age was 24 years $( \pm 5.0)$ with a range from 18 to 41 years. The three groups were comparable in their demographic characteristics and baseline SPT and TST (Table 1). Within group analyses showed no differences in the pain thresholds across the three sites. Paired t-tests indicated that SPTs were statistically significantly lower than TSTs at all three sites $(\mathrm{p}<0.001)$, indicating that temporal summation of pain was successfully induced.

\section{Temporal and spatial characteristics of the effect of acupuncture on SPT}

A multifactor ANOVA with two repeated measures (time and site) was used to analyse whether the three interventions changed SPT differently, how long the effect lasted and how widely the effect spread. Statistically significant interactions of intervention group by time $\left(F_{4,132}=9.5, p<0.001\right)$, intervention group by site $\left(F_{4,132}=4.9, p=0.002\right)$, and intervention group, time by site $\left(\mathrm{F}_{8,132}=2.5, \mathrm{p}=0.016\right)$ were identified.

One-way ANOVAs were then used to analyse SPT measured at two time points (30 minutes after treatment and 24 hours after) and three sites, respectively. Post hoc analyses were conducted with Bonferroni corrections to identify differences between intervention groups. As illustrated in Figure 3, SPT of the MA and EA groups increased immediately after the treatment and the analgesic effects became stronger 24 hours after the 
intervention. However only SPTs measured on the non-treatment leg at 24 hours $(\mathrm{p}=$ $0.015)$ and treatment leg at 30 minutes after treatment $(\mathrm{p}=0.036)$ and 24 hours $(\mathrm{p}=$ 0.009) in the EA group were statistically significantly higher than those in SA. There was no difference between MA and EA or MA and SA.

\section{Temporal and spatial characteristics of the effect of acupuncture on TST}

A multifactor ANOVA with two repeated measures (time and site) was used to analyse TST. Statistical significant interactions of intervention groups and time $\left(\mathrm{F}_{4,132}=22.0, \mathrm{p}<\right.$ $0.001)$, intervention groups and site $\left(\mathrm{F}_{4,132}=5.2, \mathrm{p}=0.001\right)$, and intervention, time and site $\left(\mathrm{F}_{8,132}=3.2, \mathrm{p}=0.002\right)$ were identified.

Similarly, TST in the MA and EA groups increased immediately after the intervention and the analgesic effects became stronger 24 hours after the intervention as illustrated in Figure 4. TST in the EA group, measured on the treatment leg 30 minutes and 24 hours after the intervention and on non-treatment leg 24 hours were statistically significantly higher than those in SA (ANOVA, $\mathrm{p}=0.038,0.006$ and 0.003 , respectively). There was no statistically significant difference in TST between MA and EA or MA and SA.

\section{Changes in SPT versus TST in the EA group}

To investigate if the changes in SPT and TST differ, a multifactor ANOVA with threerepeated measures (site, time, type of pain threshold) was used to analyse percentage changes of SPT and TST of the EA group. There was no main effect of type of pain threshold $\left(\mathrm{F}_{1,11}=1.514, \mathrm{p}=0.144\right)$; and were trends for time by type $\left(\mathrm{F}_{1,11}=4.189, \mathrm{p}=\right.$ $0.065)$ and site by time by type $\left(\mathrm{F}_{1,11}=4.639, \mathrm{p}=0.054\right)$. Two-way ANOVAs (time, types of pain threshold) were used to analyse data of treatment and non-treatment legs, 
indicating a trend for statistical significant interaction of time by type on the nontreatment leg $\left(\mathrm{F}_{1,11}=4.325, \mathrm{p}=0.062\right)$. TST potentially increased more quickly at 24 hours when compared to SPT.

Table 2 illustrates the spatial distribution of percentage changes of SPT and TST in the EA group. Overall, the increase in both SPT and TST was higher on the treatment leg than non-treatment leg. The effect was less strong on the forearm of the non-treatment site.

\section{Other outcome assessments}

A one-way ANOVA analysis showed no difference among the three groups in SSAI score measured after the interventions (mean \pm SD: SA $30.4 \pm 6.6$; MA $36.0 \pm 12.4$ and EA $\left.30.0 \pm 6.8 ; \mathrm{F}_{2,35}=1.64, \mathrm{p}=0.205\right)$.

Table 3 presents the pain rating by the participants in the three groups. There was no group difference. In general, more participants in the EA and MA reported that acupuncture was painful than those in SA did. No participants reported severe pain to acupuncture stimulation or any common side effects such as fatigue or dizziness during or after the intervention.

Nineteen out of 36 participants thought they had real acupuncture and the rest did not know. None of the participants thought they had sham acupuncture. No statistically significant difference was detected among the three groups, indicating the blinding was $\operatorname{successful}\left(\chi_{2}^{2}=0.223, \mathrm{p}=0.895\right)$. 


\section{Discussion}

To our knowledge, this is the first study that examines the effect of acupuncture on temporal summation of pain in humans. Our results indicate that EA of 2/100 Hz induces a significant analgesic effect expressed mainly in the same spinal segment of the acupuncture site and the effect becomes more potent 24 hours after the intervention. In addition, we found no significant differences in the changes of SPT and TST in the EA group, indicating a similar mechanism underlying the analgesia of EA on these two types of pain threshold.

\section{Trial design}

The present study adopted a well-accepted trial procedure with successful allocation concealment and blinding. A non-invasive SA procedure, modified from that employed in a previous trial (Lao et al. 1999), was successfully implemented. The SA had little impact on SPT or TST. Moreover, participants in the SA group were not aware they had been given a sham procedure. This participant-assessor blinded design ensures that performance bias was controlled.

Being an invasive procedure, acupuncture can produce pain, anxiety and stress. Previous studies had shown that anxiety or stress increases or decreases pain threshold (Cornwall and C. 1988; Cathcart et al. 2008; Dufton et al. 2008; Alghamdi and Al-Sheikh 2009; Vedolin et al. 2009). The level of anxiety in this study was measured with the wellaccepted Spielberg State Anxiety Inventory. Participants were not particularly stressed by the intervention as the mean score was below 40 (Spielberg 1989) before and after 
acupuncture, indicating that the increased pain thresholds were not due to the stress associated with needling.

The interpretation of the results is limited due to the small sample size, which might have increased the type II error between EA and MA or MA and SA. There was no existing data for a sample size calculation for this study. The interim data analysis was undertaken after we tested 27 subjects and it was indicated that 36 subjects would give adequate power to detect the difference between EA and SA groups. Based on the data presented in this paper, further sample size calculations indicated that 28 and 51 subjects in each group were required, respectively, to detect the difference between EA and MA in SPT and TST on the treatment leg 24 hours after the intervention with $80 \%$ power. To detect the same difference between MA and SA groups, 29 and 20 subjects in each group are needed.

The obstacles to recruitment are 1) we had to recruit acupuncture naïve participants to ensure the success of blinding; 2) participants had to visit the clinic twice within 24 hours, imposing a high demand of participants' time; 3) we did not pay or reimburse subjects' time. Due to this limitation of a relatively small sample size, the discussion will focus on the effect of EA observed in the current study.

\section{The effects of EA on SPT and TST}

SPT and TST changed in a similar manner in the EA group. Both increased immediately after intervention and continued to increase in the following 24 hours, indicating that EA does not have a specific anti-central sensitisation mechanism as some medications have. 
For instance, NMDA antagonists, such as ketamine, and anti-depressants including imipramine or venlafaxine, enhance TST but have little effect on SPT (Arendt-Nielsen et al. 1995; Enggaard et al. 2001a; Enggaard et al. 2001b).

Our results indicate that the EA analgesic effect observed is less likely to be mediated via its inhibition on NMDA receptors. Recent animal studies supported the non-NMDA proposition. One study reported that ketamine $(0.5 \mathrm{mg} / \mathrm{kg})$ not only did not compete against $100 \mathrm{~Hz}$ EA, but also enhanced the effect of EA. Rats treated with both ketamine and EA had a much higher pain tolerance threshold (Huang et al. 2005). Similarly, EA of $2 \mathrm{~Hz}$ on ST36 combined with a low-dose ketamine, which was not effective on its own, attenuated allodynia in a spinal never ligation model (Huang et al. 2004). It appears that NMDA antagonists and EA of either high or low frequency are not competitive, but complementary in their analgesic effect.

EA effect is similar to that of codeine, which increases both SPT and TST (Enggaard et al. 2001b). Codeine binds to $\mu$ and $\delta$ opioid receptors (Mignat et al. 1995) to exert its analgesic effects. Previous animal and human studies have shown that $2 / 100 \mathrm{~Hz}$ EA enhances the release of $\beta$-endorphins, enkephalins and dynorphins into the cerebrospinal fluid (Han et al. 1991; Chen et al. 1992). These endogenous opioid peptides bind to $\mu, \delta$ and $\kappa$ receptors, respectively. Consequently, EA may have some non-specific inhibitory effects on windup in the CNS through activation of endogenous opioids. The exact neural mechanism of how EA inhibits temporal summation of pain is not clear. Given TST increased on both legs, the central mechanism is likely involved. However the 
involvement of peripheral mechanisms, such as release of endogenous opioid peptides (Zhang et al. 2005) at the peripheral site upon EA may also contribute to the increased SPT and TST on the treatment leg.

\section{The temporal and spatial characteristics of acupuncture analgesia}

In the present study, the analgesic effect induced by EA on the treatment sites appeared within 30 minutes after the intervention and lasted for at least 24 hours, suggesting that EA of 2/100 may have a dual action, possibly, a quick-onset neural action and a longlasting neural-humeral action. These findings reinforce the current understanding that high frequency EA activates large-myelinated fibres while low frequency EA triggers the releases of endogenous opioids (Han 1989), inducing a slow acting mechanism via the neuro-humoral mechanism.

Clinically, after a single acupuncture treatment, patients often report beneficial effect overnight or in a couple of days (Mann 2000). In an animal study, mRNA gene transcription of preproenkephalin, the precusor of one type of endogenous opioid, enkephalin, gradually increased in the brain and peaking at 48 hours, and then a $50 \%$ decline after 72 hours (Guo et al. 1996a). Future studies need to examine how long EA analgesia after one session of treatment can last in humans.

The present study also showed that the analgesic effect of EA was mainly segmentally distributed, and is different from the results reported by Zaslawski and colleagues (2003). Manually needling Hegu (LI4) on the right hand was found to increase mechanical pressure pain thresholds at 10 body sites on the arm, trunk, face and legs without 
following any particular patterns. Similarity, manual needling of Hegu (LI4) and Shousanli (LI10) on the left forearm also showed an increase in electrical pain threshold measured at 12 sites over the body (Chiang et al. 1973). Both studies demonstrated a widespread effect of acupuncture analgesia.

The plausible explanations for the inconsistent findings between our and the other two studies may be due to: 1) types of interventions; 2) types of pain threshold; and 3) acupoints used.

Firstly, MA was used in Zaslwaski's study, whereas EA and MA were used in the current study. For MA, we adopted the same needling techniques as described by Zaslwaski and colleagues (2003). Similar to EA, the MA group demonstrated a segmental effect. This suggests that the discrepancy is unlikely due to the stimulation mode.

Secondly, pressure pain thresholds were tested in Zaslwaski's study. In the present study, electrical pain threshold is measured. However, Chiang and colleagues also tested electrical pain threshold (1973) and reported a widespread effect similar to that of Zaslwaski's study. Therefore, types of pain thresholds tested might not be the answer to the question

Finally, both Zaslwaski's and Chiang's studies used LI4 for acupuncture, whereas ST36 and ST40 on the leg were needled in the present study. LI4 and ST36 are acupoints showing strong analgesic effects (Han 2004); however, direct comparison of the two 
acupoints has not been undertaken. It is possible that LI4 has more potent and widespread effect than ST36 does for pain reduction.

\section{The analgesic effects of different modes of acupuncture}

Findings from this study showed that EA exerted a quicker and stronger effect than MA did. The enhanced SPT and TST in the EA group cannot be explained by possible changes in skin impedance upon electrical stimulation, which in turn reduces skin conduction and falsely enhances cutaneous pain thresholds. Recent studies show that EA does not affect skin impedance either at the site of acupuncture (Leung et al. 2006) or the non-treatment side (Kim et al. 2009). In addition, a constant current unit was used in our study to ensure that skin impedance did not impact on the intensity of stimulation (Walsh 2008). Furthermore, such a change does not explain why SPT and TST on the opposite leg, where no EA was delivered, also increased at 24 hours.

The different analgesia induced by EA and MA seen this study is supported by available clinical evidence (Tsui and Leung 2002; Inoue et al. 2008). However the difference in durations of stimulation could have contributed to this outcome. In the present study, the total stimulation time of the two acupoints was 60 seconds in the MA group and 25 minutes in the EA group. Thus, caution should be taken when interpreting the findings. Indeed, when the stimulation time of the two modes were comparable, no differences were found between EA and MA (Kong et al. 2005). 
The lack of difference between the MA and SA groups in this study is likely a type II error due to a small sample size explained previously. 


\section{Conclusions}

EA of 2/100 Hz produces a potent analgesic effect measured with SPT and TST at 24 hours after a single session of treatment. EA is not more potent on TST than on SPT. The effect is likely to be mediated via a quick-onset neural mechanism and a long-lasting neural-humoral mechanism and is limited to the bilateral segment of the needling sites.

The anti-temporal summation mechanisms of acupuncture may be elucidated by further studies using various agents, such as NMDA and opioid receptor antagonists. Future human studies should have larger sample sizes, and using heat and mechanical temporal summation models to verify the current results in patients with pain. 


\section{Conflicts of interest}

We have no conflicts of interest to declare. 


\section{Acknowledgments}

We thank all the volunteers for their time and efforts. This project was partially supported by a research grant from the Australian Acupuncture and Chinese Medicine Association

Ltd. We also would like to thank Mr Linghan Bai for his drawing. 


\section{References}

Alghamdi KS, Al-Sheikh MH. Effect of stress on pain perception in young women. Saudi Med J 2009;30(4):478-484.

Arendt-Nielsen L, Brennum J, Sindrup S, Bak P. Electrophysiological and psychophysical quantification of temporal summation in the human nociceptive system. Eur J Appl Physiol Occup Physiol 1994;68:266-273.

Arendt-Nielsen L, Pertersen-Feilx S. Wind-up and neuroplasticity: is there a correlation to clinical pain? Eur J Anaesthesiol 1995;12(suppl. 10):1-7.

Arendt-Nielsen L, Petersen-Felix S, Fischer M, Bak P, Bjerring P, Zbinden AM. The effect of N-Methyl-Daspartate antagonist (Ketamine) on single and repeated nociceptive stimuli: a placebo-controlled experimental human study. Anesth Analg 1995;81:63-68.

Berman BM, Lao L, Langenberg P, Lee WL, Gilpin AM, Hochberg MC. Effectiveness of acupuncture as adjunctive therapy in osteoarthritis of the knee: a randomized, controlled trial. Ann Intern Med 2004;141(12):901-910.

Cathcart S, Petkov J, Pritchard D. Effects of induced stress on experimental pain sensitivity in chronic tension-type headache sufferers. Eur J Neurol 2008;15(6):552-558.

Chen XH, Han JS, al e. Analgesia induced by electroacupuncture of different frequencies is mediated by different types of opioid receptors: another cross-tolerance study. Behav Brain Res 1992;47(2):143-149.

Chiang CY, Chang CT, Chu HC, Yang LF. Peripheral afferent pathway for acupuncture analgesia. Scientia Sinica 1973;16(2):210-217.

Cornwall A, Donderi DC. The effect of experimentally induced anxiety on the experience of pressure pain. Pain 1988;35(1):105-113.

Dufton LM, Konik B, Colletti R, Stanger C, Boyer M, Morrow S, Compas BE. Effects of stress on pain threshold and tolerance in children with recurrent abdominal pain. Pain 2008;136(1-2):38-43.

Enggaard TP, Klitgaard NA, Gram LF, Arendt-Nielsen L, Sindrup SH. Specific effect of venlafaxine on single and repetitive experimental painful stimuli in humans. Clin Pharmacol Ther 2001a;S69(4):245-251.

Enggaard TP, Poulsen L, Arendt-Nielsen L, Hansen SH, Bjornsdottir I, Gram LF, Sindrup SH. The analgesic effect of codeine as compared to imipramine in different human experimental pain models. Pain 2001b;92(1-2):277-282.

Fink M, Rosted P, Bernateck M, Stiesch-Scholz M, Karst M. Acupuncture in the treatment of painful dysfunction of the temporomandibular joint -- a review of the literature. Forsch Komplementarmed 2006;13(2):109-115.

Furlan AD, van Tulder MW, Cherkin DC, Tsukayama H, Lao L, Koes BW, Berman BM. Acupuncture and dry-needling for low back pain (review) Cochrane Database Syst Rev 2005(1).

Green S, Buchbinder R, Hetrick S. Acupuncture for shoulder pain (review). Cochrane Database Syst Rev 2005(2):CD005319.

Guo HF, Cui X, Hou Y, Tian J, Wang X, Han J. C-Fos proteins are not involved in the activation of preproenkephalin gene expression in rat brain by peripheral electric stimulation (electroacupuncture). Neurosci Lett 1996a;207(3):163-166.

Guo HF, Tian J, Wang X, Fang Y, Hou Y, Han J. Brain substrates activated by electroacupuncture (EA) of different frequencies (II): Role of Fos/Jun proteins in EA-induced transcription of preproenkephalin and preprodynorphin genes. Res Mol Brain Res 1996b;43(1-2):167-173.

Han JS. Central neurotransmitters and acupuncture analgesia. In: B Pomeranze, G Stux, editors. Scientific Bases of Acupuncture. Berlin: Springer-Verlag, 1998, 7-34..

Han JS. Acupuncture and endorphins. Neuroscience Letters 2004;361:258-261.

Han JS, Chen XH, Sun SL, Xu XJ, Yuan Y, Yan SC, Hao JX, Terenius L. Effect of low- and highfrequency TENS on Met-enkephalin-Arg-Phe and dynorphin: A immunoreactivity in human lumbar CSF. Pain 1991;47(3):295-298

Huang C, Li H-T, Shia Y-S, Han J-S, Wan Y. Ketamine potentiates the effect of electroacupuncture on mechanical allodynia in a rat model of neuropathic pain. Neurosci Lett 2004;368:327-331. 
Huang C, Long H, Shi YS, Han JS, Wan Y. Ketamine enhances the efficacy to and delays the development of tolerance to electroacupuncture-induced antinociception in rats. Neurosci Lett 2005;375(2):138142.

Inoue M, Kitakoji H, Yano T, Ishizaki N, Itoi M, Katsumi Y. Acupuncture treatment for low back pain and lower limb symptoms-the relation between acupuncture or electroacupuncture stimulation and sciatic nerve blood flow. Evid Based Complement Alternat Med 2008;5(2):133-143.

Kim MS, Cho YC, Moon JH, Pak SC. A characteristic estimation of bio-signals for electro-acupuncture stimulations in human subjects. Am J Chin Med 2009;37(3):505-517.

Kong J, Fufa DT, Gerber AJ, Rosman IS, Vangel MG, Gracely RH, Gollub RL. Psychophysical outcomes from a randomized pilot study of manual, electro, and sham acupuncture treatment on experimentally induced thermal pain. J Pain 2005;6(1):55-64.

Lao LX, Hamilton SG, R., Langenberg P, Berman B. Evaluation of acupuncture for pain control after oral surgery: a placebo-controlled trial. Arch Otolaryngol Head Neck Surg 1999;125(5):567-572.

Lee TL. Acupuncture and chronic pain management. Ann Acad Med Singapore 2000;29(1):17-21.

Leung AY, Park J, Schulteis G, Duann JR, Yaksh T. The electrophysiology of de qi sensations. J Altern Complement Med 2006;12(8):743-750.

Mann F. Reinventing Acupuncture: a New Concept of Ancient Acupuncture. Oxford: ButterworthHeinemann, 2000.

Martin DP, Sletten CD, Williams BA, Berger IH. Improvement in fibromyalgia symptoms with acupuncture: results of a randomized controlled trial. Mayo Clinic Proceeding 2006;81(6):749-757.

Melchart D, Linde K, Berman BM, White A, Vickers A, Allais G, Brinkhaus B. Acupuncture for idiopathic headache. . Cochrane Database Syst Rev 2001(1).

Mignat C, Wille U, Ziegler A. Affinity profiles of morphine, codeine, dihydrocodeine and their glucuronides at opioid receptor subtypes. Life Sci 1995;56(10):793-799.

Petersen-Felix S, Arendt-Nielsen L, Bak P, Fischer M, Bjerring P, Zbinden AM. The effect of isoflurane on repeated nociceptive stimuli (central temporal summation). Pain 1996;64:277-281.

Price DD, Hayes RL, Ruda M, Dubner R. Spatial and temporal transformation of input to spinothalamic tract neurons and their relation to somatic sensations. J Neurophysiol 1978;41:933-947.

Price DD, Mao J, Frenk H, Mayer DJ. The N-methyl-D-aspartate receptor antagonist dextromethorphan selectively reduces temporal summation of second pain in man. Pain 1994;59:165-174.

Spielberg CD. State-Trait Anxiety Inventory: A comprehensive bibliography Palo Alto, CA: Consulting Psychologists Press, 1989.

Spielberg CD, Gorsuch RL, Lushene PR, Vagg PR, Jacobs GA. State-Trait Anxiety Inventory. California: Mind Garden., 1983.

Trinh KV, Graham N, Gross AR, Goldsmith CH, Wang E, Cameron ID, Kay T, Group CO. Acupuncture for neck disorders (review). Cochrane Database Syst Rev 2006(3).

Tsui P, Leung MC. Comparison of the effectiveness between manual acupuncture and electro-acupuncture on patients with tennis elbow. Acupunct Electrother Res 2002;27(2):107-117.

Urban MO, Gebhart GF. Central mechanisms in pain. Med Clin Noth Am 1999;83(3):585-596.

Vedolin GM, Lobato VV, Conti PCR, Lauris JRP. The impact of stress and anxiety on the pressure pain threshold of myofascial pain patients. J Oral Rehabil 2009;36(5):313-321.

Walsh D. Introduction to low frequency currents. In: T Watson, editor. Electrotherapy: Evidence-Based Practice. Elsevier Churchill Livingstone, 2008; 203-210.

Woolf CJ, Thompson SW. The induction and maintenance of central sensitization is dependent on Nmethyl-D-aspartic acid receptor activation: implications for the treatment of post-injury pain hypersensitivity states. Pain 1991;44(3):293-299.

Xue CCL, Dong L, Polus B, English RA, Zheng Z, Da Costa C, Story D. Electroacupuncture for tensiontype headache on distal acupoints only: a randomized, controlled, crossover trial. Headache 2004;44(4):333-341.

Zaslawski CJ, Cobbin D, Lidums E, Petocz P. The impact of site specificity and needle manipulation on changes to pain pressure threshold following manual acupuncture: a controlled study. Complement Ther Med 2003;11(1):11-21.

Zhang GG, Yu CS, Lee WL, Lao LX, Ren K, Berman BM. Involvement of peripheral opioid mechanisms in electroacupuncture analgesia. Explore 2005;1(5):365-371. 
Zheng Z, Gou RXJ, Helme RD, Muir A, Da Costa C, Xue CCL. The effect of electroacupuncture on opioid-like medication consumption by chronic pain patients: A pilot randomized controlled clinical trial Eur J Pain 2008;12(5):671-676. 
Tables 1-4

Table 1 Demographic characteristics and baseline data of each group [No of count or Mean (SD)].

\begin{tabular}{llccc}
\hline & SA $(\boldsymbol{n}=\mathbf{1 2})$ & MA $(\boldsymbol{n}=\mathbf{1 2})$ & EA $(\boldsymbol{n}=\mathbf{1 2})$ \\
\hline Age (years) & $24.4(4.4)$ & $24.0(4.3)$ & $23.6(6.4)$ \\
\hline Gender (Male : Female) & $5: 7$ & $7: 5$ & $9: 3$ \\
\hline $\begin{array}{l}\text { Dominant hand } \\
\text { (Right }: \text { Left) }\end{array}$ & $11: 1$ & $12: 0$ & $10: 2$ \\
\hline SSAI & & $32.7(7.3)$ & $31.4(7.7)$ & $29.2(8.5)$ \\
\hline $\begin{array}{l}\text { SPT } \\
(\mathrm{mA})\end{array}$ & Treatment leg & $2.0(0.6)$ & $2.0(0.8)$ & $1.8(0.8)$ \\
\cline { 2 - 5 } & Non-treatment leg & $2.1(0 . .8)$ & $1.9(0.9)$ & $2.0(0.8)$ \\
\cline { 2 - 5 } & forearm & $2.3(0.8)$ & $1.9(0.9)$ & $2.0(0.8)$ \\
\hline $\begin{array}{l}\text { TST } \\
(\mathrm{mA})\end{array}$ & Treatment leg & $1.3(0.5)$ & $1.2(0.7)$ & $1.2(0.4)$ \\
\cline { 2 - 5 } & Non-treatment leg & $1.3(0.5)$ & $1.1(0.7)$ & $1.2(0.5)$ \\
\cline { 2 - 5 } & forearm & $1.3(0.6)$ & & $1.1(0.5)$ \\
\hline
\end{tabular}

Table 2 Percentage changes of SPT and TST in the EA group $(n=12)$ at three sites and two time points.

\begin{tabular}{l|llll}
\hline & \multicolumn{2}{|c}{ SPT } & \multicolumn{2}{c}{ TST } \\
\hline & 30 minutes after & 24 hours after & 30 minutes after & 24 after \\
\hline Treatment leg & 85 & 154 & 67 & 90 \\
\hline $\begin{array}{l}\text { Non-treatment } \\
\text { leg }\end{array}$ & 45 & 77 & 36 & 112 \\
\hline Forearm & 37 & 50 & 33 & 73 \\
\hline
\end{tabular}


Table 3 Number of participants who rated the intensity of acupuncture stimulation

\begin{tabular}{|c|c|c|c|c|c|}
\hline & \multicolumn{3}{|c|}{ The intensity of acupuncture stimulation } & \multirow[b]{2}{*}{$\chi^{2}(d f)$} & \multirow[b]{2}{*}{$P^{*}$} \\
\hline & No pain & $\begin{array}{l}\text { Slight/mild } \\
\text { pain }\end{array}$ & Moderate pain & & \\
\hline $\mathrm{SA}(n=12)$ & 7 & 4 & 1 & $9.5(4)$ & 0.110 \\
\hline $\mathrm{MA}(n=12)$ & 2 & 5 & 5 & & \\
\hline $\mathrm{EA}(n=12)$ & 2 & 6 & 4 & & \\
\hline
\end{tabular}

$d f$ : degrees of freedom.

Table 4 Participants' perception of acupuncture.

\begin{tabular}{|c|c|c|c|c|c|}
\hline & \multicolumn{3}{|c|}{ Volunteers'perception of treatment } & \multicolumn{2}{|c|}{ Statistical test } \\
\hline & $\begin{array}{c}\text { Real } \\
\text { Acupuncture }\end{array}$ & $\begin{array}{c}\text { Placebo/sham } \\
\text { acupuncture }\end{array}$ & Don't know & $\chi^{2}(d f)$ & $p$ \\
\hline SA $(n=12)$ & 7 & 0 & 5 & $0.223(2)$ & 0.895 \\
\hline $\mathrm{MA}(\mathrm{n}=12)$ & 6 & 0 & 6 & & \\
\hline $\mathrm{EA}(\mathrm{n}=12)$ & 6 & 0 & 6 & & \\
\hline
\end{tabular}


Figures 1-4 Titles

Figure 1 Sites of acupuncture treatment and the assessment of pain threshold. (black dots: site of acupuncture treatment; filled elongated circles: sites at which SPT and TST were assessed).

Figure 2 The trial procedure.

Figure 3a-c Single pain thresholds (SPT, Mean and SEM) of the three intervention groups tested at the three body sites at baseline, immediately and 24-hours after the interventions $(n=12$ in each group). $* \mathrm{p}<0.05$ when compared EA with SA with Bonferroni corrections.

Figure 4a-c Temporal summation thresholds (TST, Mean and SEM) of the three intervention groups tested at the three body sites at baseline, immediately and 24-hours after the interventions ( $\mathrm{n}=12$ in each group). $* \mathrm{p}<0.05$ when compared EA with SA with Bonferroni corrections. 


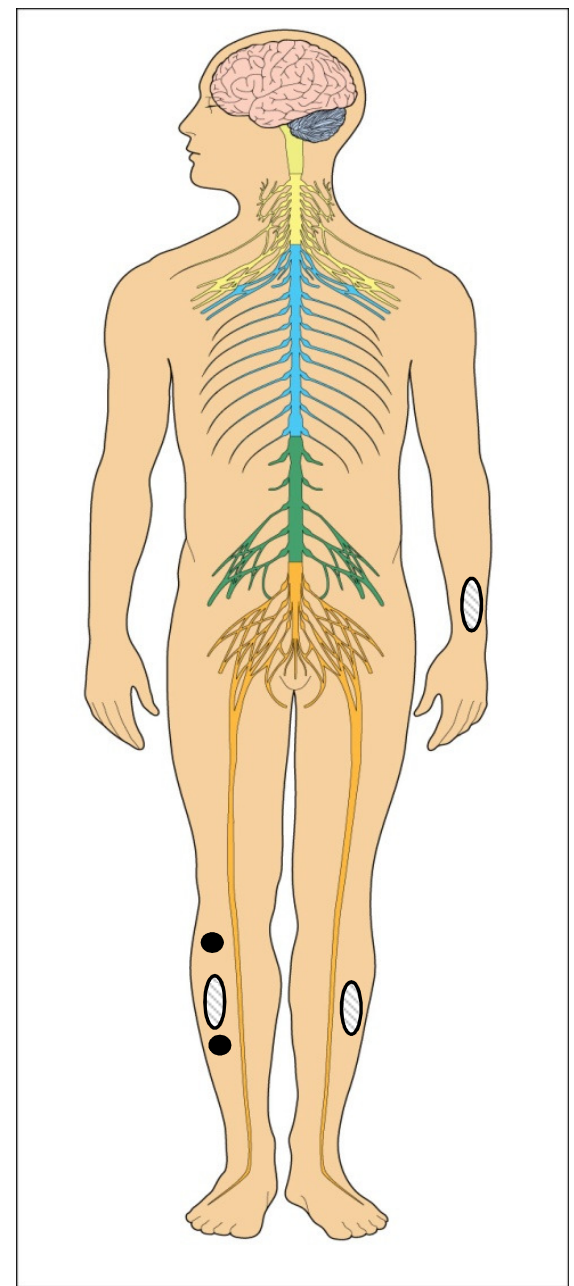

Figure 1 Sites of acupuncture treatment and the assessment of pain threshold.

(black dots: site of acupuncture treatment; filled elongated circles: sites at which SPT and TST were assessed). 


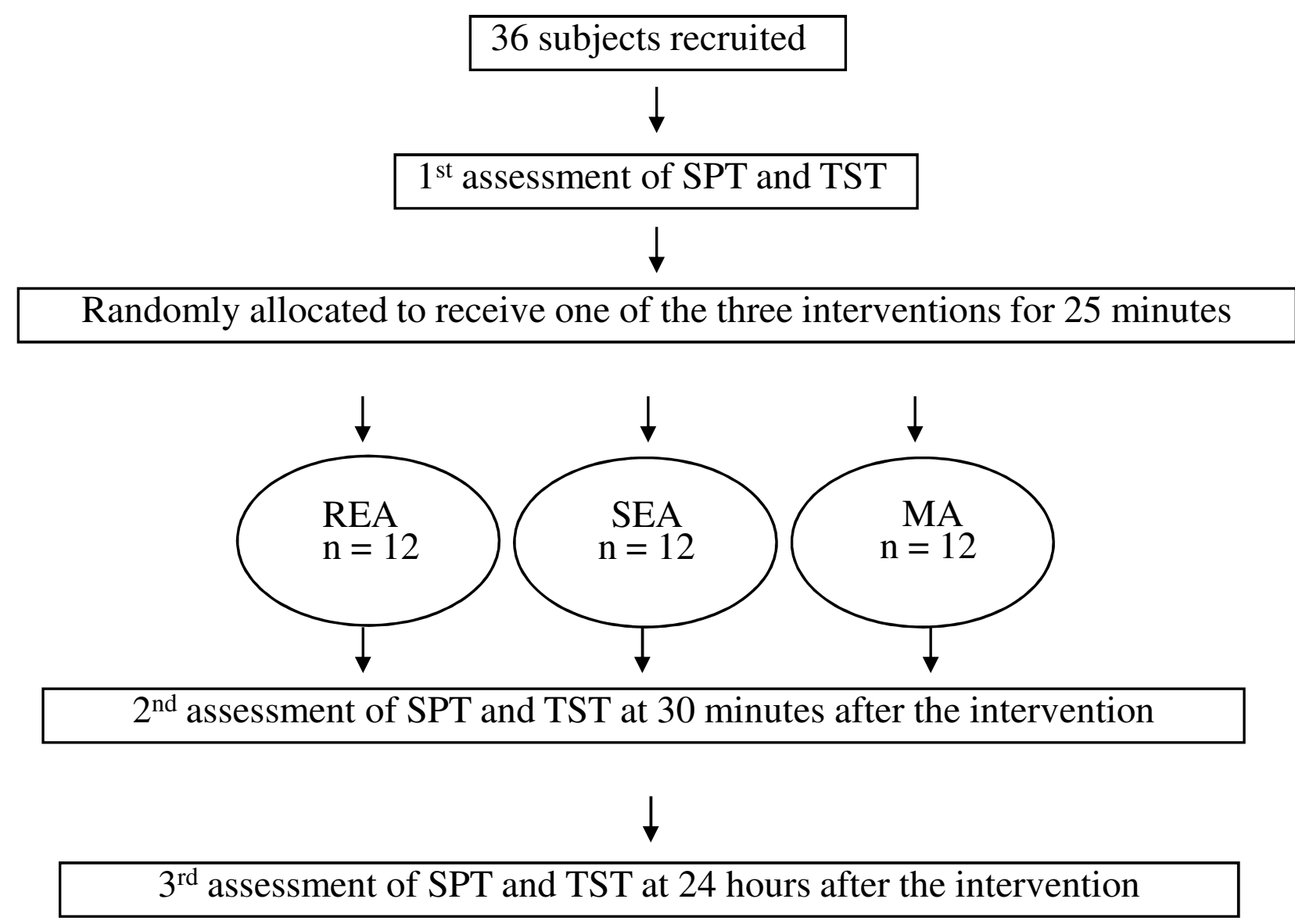

Figure 2 Trial procedure. 


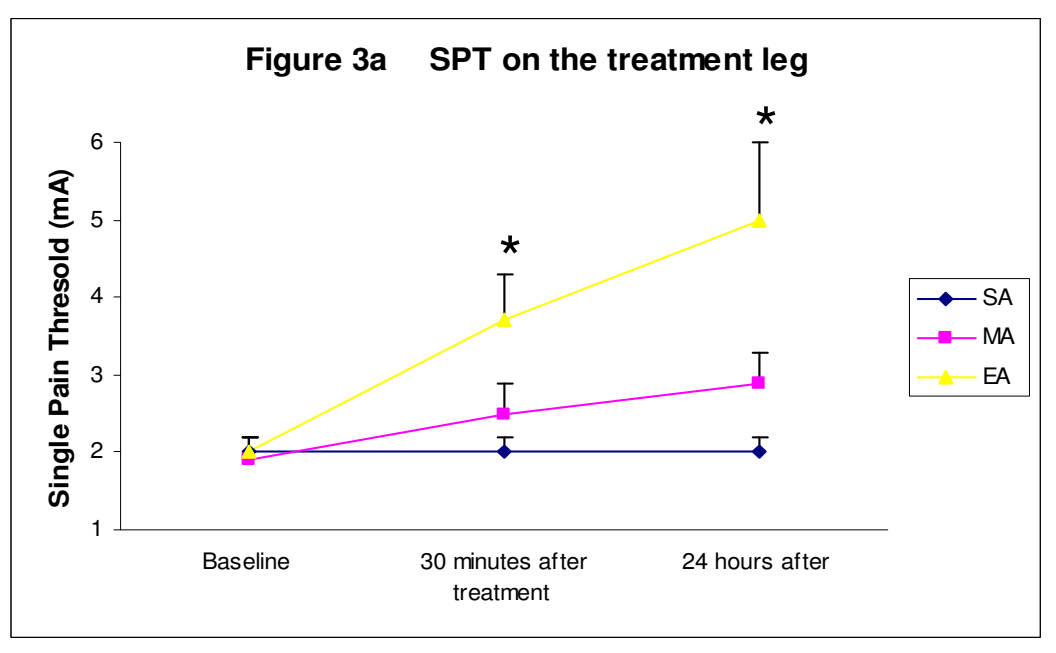




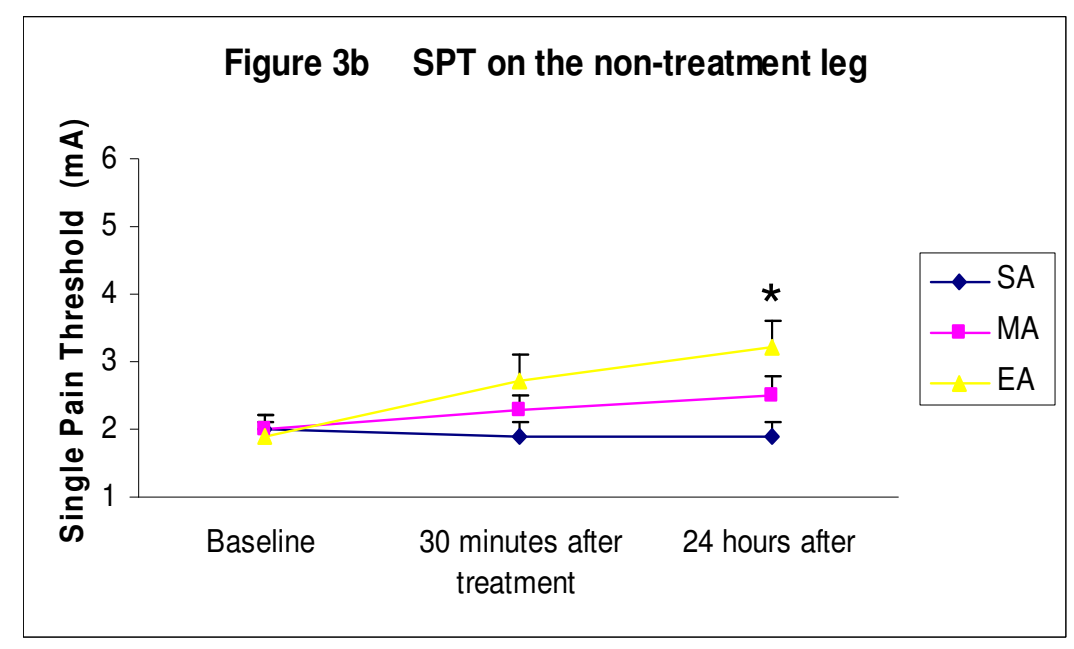




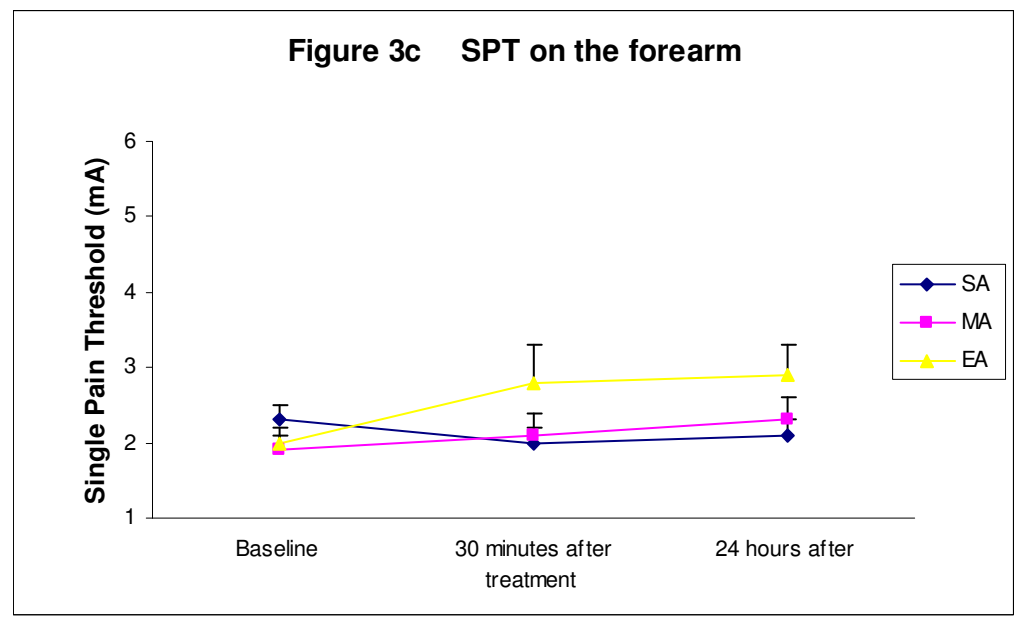

Figure 3a-c

Single pain thresholds (SPT, Mean and SEM) of the three intervention groups tested at the three body sites at baseline, immediately and 24-hours after the interventions ( $n=12$ in each group). ${ }^{*} p<0.05$ when compared EA with SA with Bonferroni corrections. 


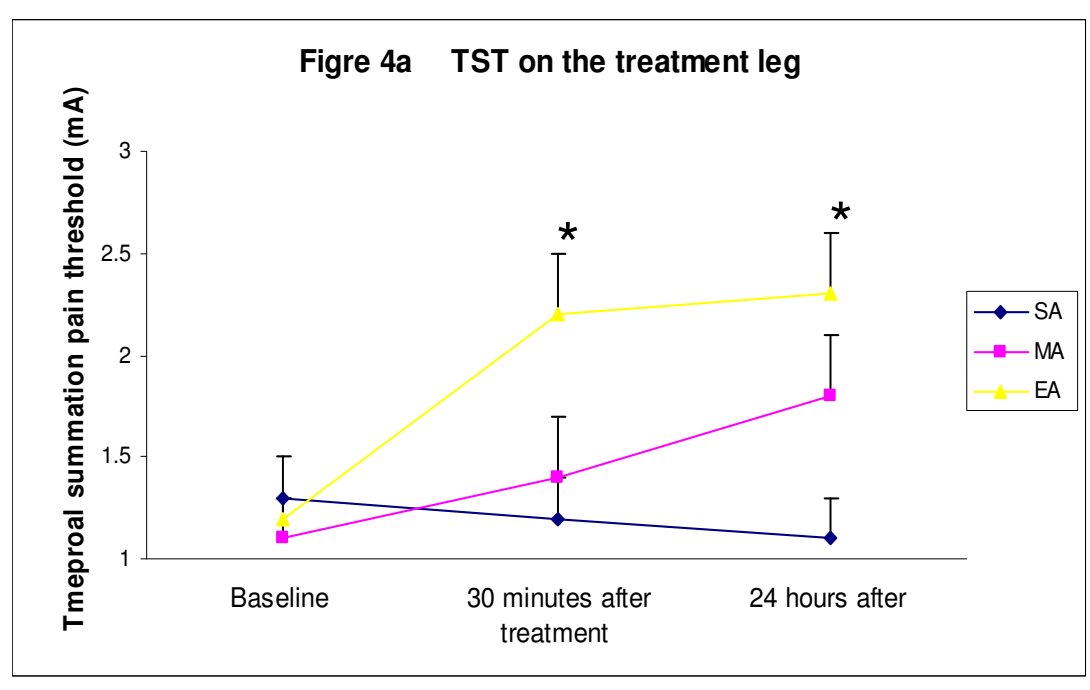




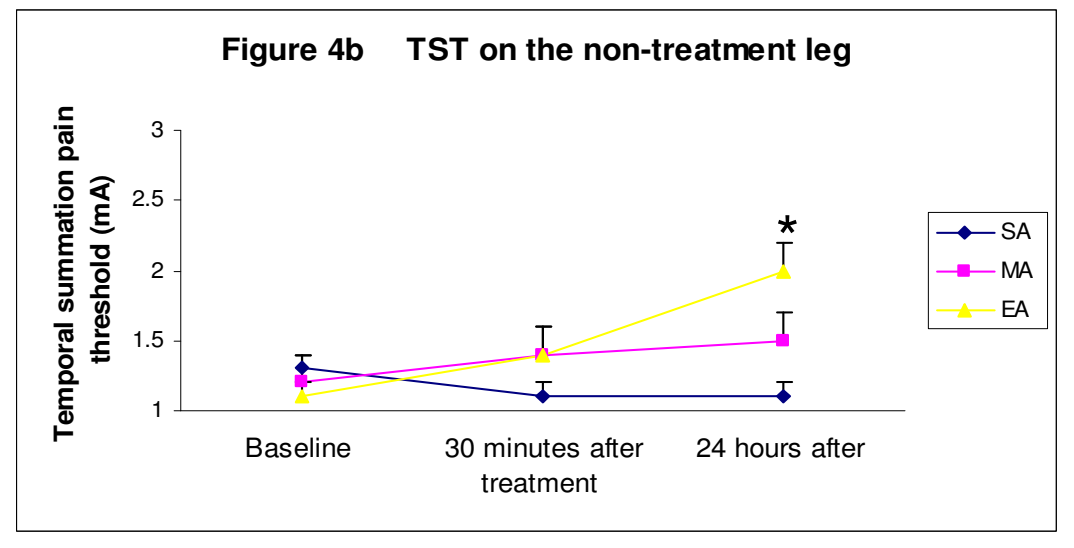




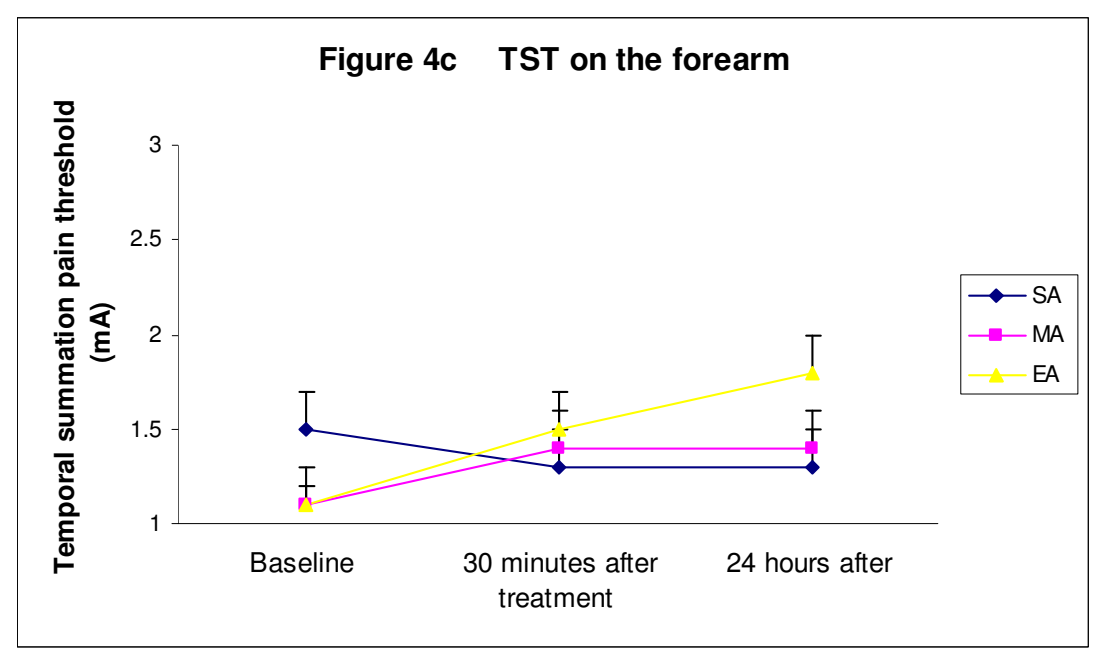

Figure 4a-c

Temporal summation thresholds (TST, mean and SEM) of the three intervention groups tested at the three body sites at baseline, immediately and 24-hours after the interventions ( $n=12$ in each group). ${ }^{*} p<0.05$ when compared EA with SA with Bonferroni corrections. 